\title{
LA BIODIVERSIDAD COMO REFERENTE PARA PROMOVER LA CONSERVACIÓN DE LOS ECOSISTEMAS COLOMBIANOS
}

\author{
BIODIVERSITY AS A REFERENCE TO PROMOTE THE \\ CONSERVATION OF COLOMBIA'S ECOSYSTEMS
}

\author{
Alejandro Henao-Castro* \\ Recibido: Junio 15, 2021 \\ Aceptado: Octubre 13, 2021
}

* Magíster en Ciencias
Marinas de la Universidad de
Bogotá Jorge Tadeo Lozano.
Estudiante de Doctorado en
Educación y Cultura Ambiental
Universidad Surcolombiana,
Colombia. Docente de la
Institución Educativa Luis
Carlos López, Cartagena de
Indias (Colombia).
henaocastro.alejandro@
gmail.com
0000-0002-4125-765X

Cómo citar este artículo: Henao, C. A. (2021). La biodiversidad como referente para promover la conservación de los ecosistemas colombianos. Revista PACA 11, pp. 215228.
Resumen: La biodiversidad, más que un concepto, puede entenderse como la suma de distintos componentes que no solo se limitan al número de especies en un sitio determinado, sino que también deben considerarse las condiciones imperantes en ese sitio, así como las variaciones que hacen parte de la dinámica natural, tanto del componente biótico como abiótico en distintas escalas de tiempo y espacio. Considerando lo anterior y, a pesar de la gran importancia que representa la biodiversidad para el bienestar de la humanidad, la tendencia actual redunda en un avance acelerado y descontrolado de la pérdida de biodiversidad en todos los ecosistemas del planeta. Esta problemática, evidentemente, es el resultado de la sinergia de múltiples factores que en conjunto conducen a un panorama poco alentador. En este documento se pretende reforzar el concepto y las teorías que dieron origen a la gran biodiversidad actual, el estado de conocimiento de la biodiversidad de Colombia y, además, abordar los factores que influyen en la pérdida de biodiversidad, todo esto con el fin de considerar la educación ambiental como una herramienta poderosa para contrarrestar los efectos negativos del actual comportamiento de los seres humanos.

Palabras clave: biodiversidad, especie, ecosistemas, educación ambiental, intervención antropogénica. 


\begin{abstract}
Biodiversity, more than a concept, can be understood as the sum of different components that are not only limited to the number of species in a given place, but also the prevailing conditions of such place, as well as the variations that are part of the natural dynamics of both biotic and abiotic components on different scales of time and space. Considering the above and, despite of the great importance that biodiversity represents for the well-being of humanity, the current trend results in an accelerated and uncontrolled advance of the loss of biodiversity in every ecosystems of the planet. This problem is the result of the synergy of multiple factors, that together, lead to a less than encouraging outlook. This writing aims to reinforce the concept and theories that originated the current biodiversity, the state of knowledge of Colombia's biodiversity and, in addition, addresses the factors that influence the biodiversity loss, all of this, in order to consider environmental education as a powerful tool to counteract the negative effects of the current behavior of human beings.
\end{abstract}

Keywords: anthropic intervention, biodiversity, ecosystem, environmental education, specie.

\title{
Introducción
}

En el presente documento se pretende abordar cómo los conceptos y teorías de biodiversidad pueden usarse como referente para afrontar la problemática de la pérdida de biodiversidad por medio de la educación ambiental. Para lograr este objetivo, inicialmente se habla de los distintos significados que pueden presentarse para definir este concepto, entendiendo que este es polisémico y que, dependiendo del contexto, las definiciones pueden coexistir. Por otro lado, se mencionan y explican los tipos de biodiversidad, así como los procesos evolutivos por medio de los cuales se hace posible la existencia de un gran número de especies de manera simultánea. Seguido a esto, se profundiza sobre los determinantes de la biodiversidad y cómo son provocados por acciones antropogénicas, así como los efectos negativos que esto genera en la humanidad. Finalmente, con todo lo anterior, se analiza cómo la educación ambiental presenta un rol preponderante para contrarrestar estos determinantes ambientales desde un cambio en la cultura de la sociedad.

\section{La biodiversidad como polisemia}

En la literatura se reporta un gran número de definiciones de biodiversidad, las cuales se generan principalmente dependiendo de factores como la región, los procesos que la regulan, entre otros (Stupino 
et al., 2014; Pérez-Mesa, 2019). En general no existe un solo término, y cada vez que se pretende estandarizarlo, se genera gran controversia entre la comunidad académica de distintos campos de acción. Es así como se puede hablar de biodiversidad funcional, ecosistémica, genética o incluso, términos que incluyan todos los aspectos mencionados. Asimismo, es posible encontrar conceptos de biodiversidad que incluyan o excluyan el componente evolutivo que ha permitido la especiación (Kaennel, 1998; Nuñez et al., 2003; Martín-López et al., 2007; Hamilton, 2005).

Considerando la importancia de definir y estandarizar el concepto de biodiversidad, Halffter (1994) la define como "una medida de la heterogeneidad de un sistema. En el caso de los sistemas biológicos, la diversidad se refiere a la heterogeneidad biológica, es decir, a la cantidad y proporción de los diferentes elementos biológicos que contenga el sistema" (p. 6). Asimismo, Gaston y Spicer (2004) la definen como "la variedad de vida, y se refiere colectivamente a la variación en todos los niveles de la organización biológica" (p. 4).

Por otra parte, realizando una revisión de distintas publicaciones científicas en deferentes campos de acción, es posible notar que, tal vez, uno de los conceptos de biodiversidad más ampliamente usados, es el que se planteó en la Convención Internacional de Diversidad Biológica (Aguilera y Silva, 1997; Andrade, 2011), la cual intentó definir un concepto unificado con el fin de estandarizarlo y que sea susceptible de utilizarse en diversos campos y contextos. De este modo, se propone que "la biodiversidad es la variabilidad entre organismos vivos de todas las fuentes, incluyendo los terrestres, marinos y otros ecosistemas acuáticos y los complejos ecológicos de los que hacen parte; esto incluye diversidad dentro de especies, entre especies y de ecosistemas" (The International Convention on Biological Diversity, 2003). Con este concepto, además de reconocerse el valor intrínseco de la diversidad biológica, se reconocen otros valores (ecológicos, genéticos, sociales, económicos, científicos, educativos, culturales, recreativos y estéticos), y se declara la importancia de la diversidad biológica para la evolución y para el mantenimiento de los sistemas necesarios para la vida de la biosfera (Aguilera y Silva, 1997).

Considerando todo lo anterior, es importante aclarar que el objetivo de este trabajo no es discutir sobre cuál es el concepto adecuado, por el contrario, lo que se pretende es evidenciar que el concepto de 
biodiversidad en sí es heterogéneo y que, dependiendo del criterio del investigador y los objetivos que plantee en su estudio, puede optar por adherirse a una de las tantas posibilidades.

\section{Procesos evolutivos que conducen a la biodiversidad}

En el acápite anterior se realizó una revisión cuidadosa del concepto de biodiversidad; sin embargo, no se han explicado los procesos evolutivos que conllevan a una mayor biodiversidad. Entender los mecanismos evolutivos que actúan sobre las especies puede ser una herramienta importante para abordar el estudio de la biodiversidad. Adicionalmente, este conocimiento puede ser susceptible de emplearse dentro de la educación ambiental para mitigar o contrarrestar la degradación ecosistémica, cuya base es la pérdida acelerada de la biodiversidad por factores naturales y antropogénicos.

En consonancia con lo anterior, los procesos evolutivos podrían también ayudar a destacar la importancia del sitio o ecosistema que se esté investigando. Por ejemplo, se sabe que a mayor heterogeneidad del sustrato (un suelo irregular que presente muchos hábitats disponibles para los organismos que viven en éste), mayor número de especies puede presentarse (Smith y Smith, 2015). Estos procesos evolutivos tratan entonces de explicar la especiación, entendiéndose como el mecanismo por el cual aparece una nueva especie (Soler, 2002). Un claro ejemplo de esto es explicado por Rull (2004), quien propone que la gran biodiversidad de las Tierras Altas de Guayana se atribuye principalmente a la heterogeneidad ambiental, junto con variaciones significativas en el clima durante el Cuaternario en esta zona neotropical.

Aunque existen diversos mecanismos de especiación, los más destacados son la simpátrica, parapátrica y alopátrica. La primera implica la divergencia de algunos individuos de la población. Esta especiación no requiere una separación geográfica o física; por el contrario, se atribuye principalmente a la especialización en el aprovechamiento de algún recurso por parte de unos individuos (no todos) de la población. Esto comúnmente se conoce como especialización ecológica. Un ejemplo sencillo puede ser que algunos individuos de la población empiezan a ser seleccionados por el sexo opuesto para el apareamiento (Bennet, 1997). Otro ejemplo sencillo podría consistir en que algunos de los individuos 
cambien su hábito de apareamiento y lo hagan en épocas distintas al resto. En ambos casos, se generaría un aislamiento reproductivo y, de ser sostenido en el tiempo, podría dar origen a una nueva especie (Soler, 2002).

En cuanto a la especiación parapátrica, esta ocurre por lo general en poblaciones de especies que ocupan áreas muy extensas; de este modo, los individuos que están en los márgenes opuestos dejan de encontrarse y reproducirse con los demás y, con el paso del tiempo, si se mantiene dicho aislamiento (reproductivo), se generan dos especies distintas de esta gran población inicial (Smith y Smith, 2015). Finalmente, la especiación alopátrica consiste en la separación geográfica de una población de individuos de la misma especie, provocando así la interrupción del flujo de genes entre estas. Este tipo de especiación es tal vez la que más ejemplos o casos estudiados presenta; sin embargo, un ejemplo claro puede ser la aparición del Istmo de Panamá, que separó poblaciones de especies de los océanos Atlántico y Pacífico, generando así una diversificación de especies (Soler, 2002).

\section{Diversidad de especies en el planeta y en Colombia}

Como se ha mencionado con anterioridad, si se habla de biodiversidad es imposible evitar hablar del número de especies; por lo tanto, una pregunta que ha inquietado a los seres humanos, en especial biólogos, ecólogos y taxónomos es ¿cuántas especies existen en el planeta? Si bien se han realizado distintos acercamientos, todos coinciden en que no es tarea fácil y se quedan siempre en aproximaciones. No obstante, se sabe que los grupos taxonómicos más abundantes son los coleópteros y las plantas con 400000 y 250000 especies, respectivamente (Smith y Smith, 2015). Estos números son bastante representativos si se tiene en cuenta que el cálculo aproximado de las especies identificadas a la fecha asciende a 1,7 millones de especies, y las proyecciones menos optimistas, calculan que existen 10 millones de especies en el planeta. Esto quiere decir que aún falta más del $80 \%$ de la diversidad de especies por ser descubierta y descrita.

En el caso particular de Colombia, este es considerado un país megadiverso, puesto que con un área de 1,1 millones de $\mathrm{km}^{2}$, alberga el $10 \%$ de la biodiversidad del planeta. Su ubicación geográfica y su heterogeneidad espacial le confieren características únicas que permiten 
la explosión de diversidad biológica en su territorio marino y continental (Rangel, 2005; Andrade, 2011; Arbeláez-Cortés, 2013). Este título de país megadiverso también se atribuye a que resulta ser el cuarto país con mayor diversidad de plantas, el quinto en mamíferos, primero en cantidad de especies de aves, tercero en reptiles y segundo en anfibios, peces dulceacuícolas y mariposas.

Asimismo, cuando se habla de endemismo (especies que se encuentran en un área geográfica pequeña), Colombia también se ubica en los primeros lugares para plantas, mamíferos, aves, reptiles, anfibios y mariposas (Andrade, 2011). Finalmente, según el estado de conocimiento sobre biodiversidad de Colombia, hasta el año 2011 se reportaban para la ciencia 35.476 especies de fauna y 27.881 especies de plantas, concediéndole un lugar privilegiado entre los países más biodiversos del mundo (Andrade, 2011). Cabe anotar que estas cifras son estimaciones que probablemente se quedan cortas en un país con una historia de conflicto armado que ha impedido por décadas explorar (y explotar) zonas vírgenes con alta riqueza de especies (McClanahan et al., 2019), por lo que se presume que aún queda un porcentaje significativo de especies por ser descubiertas en el país.

\section{Determinantes ambientales de la biodiversidad}

Así como se ha hablado de la gran biodiversidad del planeta y de Colombia, se hace imperativo destacar algunas de las principales amenazas que se ciernen sobre ésta. Son muchos los determinantes de la biodiversidad, dentro de los cuales destacan la deforestación debido a la ganadería y agricultura intensivas, cultivos ilícitos y construcción de obras de desarrollo (Andrade, 2011). También se ha incrementado la invasión de especies no nativas, el cambio climático, el calentamiento global, la acidificación oceánica, la contaminación por metales pesados debido al desarrollo de la industria y el sector minero, la contaminación por microplásticos, la caza de especies silvestres, la sobreexplotación de los recursos naturales, la escorrentía continental en ecosistemas marino costeros, entre otros (Frawley et al., 2019; hukla et al., 2019; Tan et al., 2019; Yilmaz y Terzi, 2019; Bashir et al., 2020).

Para un mejor entendimiento de la situación global de la degradación de la biota del planeta, cabe destacar datos recopilados por Naranjo 
(2019) en su revisión sobre imaginarios de naturaleza y el futuro de la biodiversidad. Más de 4.000 especies han disminuido su abundancia en un $60 \%$, lo que implica un posible escenario de extinción masiva; también evidenciado en el aumento significativo de especies incluidas en los listados rojos de la Unión Internacional para la Conservación de la Naturaleza (UICN). Lo anterior, sumado a que el mismo autor destaca una disminución del $89 \%$ del índice planeta vivo para el Neotrópico entre los años 1970 y 2014. Con todo esto, es clara la urgencia de desarrollar mayor investigación relacionada con la biodiversidad en escalas globales, pero también regionales y locales. De hecho, ante este panorama desalentador, es fundamental que se incrementen esfuerzos no solo en establecer patrones de disminución de biodiversidad, sino que se deben potenciar los mecanismos necesarios para lograr caracterizar y combatir las causas (no solo las consecuencias) de este acelerado deterioro (Naranjo, 2019).

En el caso de Colombia, por décadas se ha venido evidenciando un deterioro gradual de los ecosistemas marinos, costeros y continentales. Con el fin de comprender la magnitud del problema se puede tomar como ejemplo el incremento de la tasa de deforestación entre 1987 y 2002, la cual se ubicó en 221.000 hectáreas anuales, es decir, que en menos de 20 años se perdieron 3.315.135 de hectáreas de bosque nativo en el país (Andrade, 2011). Otro ejemplo interesante sobre los efectos deletéreos de las actividades antropogénicas y el calentamiento global es la degradación de arrecifes de corales. En el Parque Nacional Natural Los Corales del Rosario se ha reportado un cambio en la estructura del ecosistema, el cual ha sufrido una mortalidad masiva de corales de gran importancia ecológica como los acropóridos y un incremento significativo de la cobertura de algas (> 50\% entre 1989 y 2010). Estos cambios tienen repercusiones negativas directas e indirectas sobre la resistencia y resiliencia del ecosistema, haciéndolo más vulnerable a los demás determinantes ambientales que forman sinergias para incrementar los efectos negativos (Restrepo y Alvarado, 2011; Cruz-García y Peter, 2015).

Por otra parte, recientemente se ha demostrado que la huella ecológica en el país se ha incrementado desde 1970 hasta 2015. Correa et al. (2020) reportaron que las regiones Caribe y Andina presentan los mayores niveles de impacto antropogénico, pero también pudieron evidenciar que existen otras zonas críticas en todo el territorio nacional (algunos sitios de las regiones Amazonía y Orinoquía). Estos impactos han 
sido atribuidos principalmente a la expansión del cultivo de palma de cera entre los años 1980 y 2007 y a la explotación de hidrocarburos entre 1985 y 1993. Con este escenario, surge una gran preocupación, puesto que en la región andina se han registrado los mayores números de endemismo en el país y, adicionalmente, allí se presentan los ecosistemas de páramos los cuales regulan el recurso hídrico que sostiene a las grandes ciudades del país. Finalmente, no se debe desconocer que la región Caribe alberga la mayoría de los humedales y ciénagas, considerados ecosistemas estratégicos por sus bondades y servicios que ofrecen a la humanidad.

\section{No se puede conservar aquello que se desconoce: el rol de la educación ambiental}

Las personas pueden caer en el error de considerar que la conservación de la biodiversidad es una obligación exclusiva del Estado y que la naturaleza debe protegerse por medio de la creación de políticas que generen marcos legales de protección de la biodiversidad. No obstante, se podría pensar que el caso de Colombia es de cierta manera contrario a esta aseveración ya que, a pesar de ser un país que cuenta con un porcentaje importante de su territorio protegido por figuras de conservación como parques nacionales naturales, santuarios de fauna y flora, reservas ecológicas, distritos especiales de manejo, entre otras, ha demostrado que por sí solas, las figuras legales de protección son insuficientes para la conservación de la biodiversidad, puesto que la tala indiscriminada de bosques, la minería ilegal, la sobrepesca, la ganadería extensiva, las construcciones de obras de desarrollo, la extracción de hidrocarburos, entre otras, arremeten despiada y libremente contra los ecosistemas que albergan esa biodiversidad que tanto enorgullece al pueblo colombiano (Andrade, 2011).

Con lo anterior, se puede argumentar que la educación ambiental podría entonces considerarse una opción viable para complementar la protección legal de los sitios de mayor biodiversidad del planeta. A partir de la comprensión de los conceptos, teorías y estado de conocimiento de la biodiversidad, se contaría con insumos suficientes para promover una cultura del cuidado y protección del ambiente. Globalmente se ha aceptado y demostrado que la divulgación de la información en conjunto con la educación ambiental resulta en un arma poderosa para promover iniciativas, como es el caso de la conservación de ecosistemas y su biodiversidad (Martínez, 2010; Saito, 2013). 
Así es posible entonces generar en las personas que habitan sitios con alta biodiversidad una razón más para proteger y cuidar lo que a la evolución le ha tomado millones de años realizar. Un ejemplo de esto pueden ser las Islas Galápagos en Ecuador. Dentro de la población de esta zona del mundo, desde 1999 se vienen desarrollando diferentes estrategias de educación ambiental dirigidas a todos los sectores socioeconómicos presentes.

Dentro de las estrategias implementadas llama la atención la inclusión de la importancia de la biodiversidad y los procesos evolutivos que tuvieron lugar en la zona dentro de los currículos de los diferentes niveles académicos. A partir de la sensibilización de los pobladores locales sobre la gran riqueza biológica que tienen, se promueve el cuidado y protección de la biota presente. Asimismo, son los pobladores quienes posteriormente transmiten la importancia de la protección de la zona (por los procesos evolutivos que han ocurrido allí y la explosión de biodiversidad que esto ha generado) hacia los visitantes de otras regiones del planeta (Arciniegas y Torres, 2007).

Adicionalmente, es interesante como Van Weelie y Wals (2002) plantean que la biodiversidad puede convertirse en un vehículo interesante para unir la ciencia y la sociedad. Es decir, que la información que se deriva de la ciencia, en este caso concerniente a la biodiversidad, sus teorías, conceptos y los procesos que la originan, pueden ser adoptados por la sociedad para un bien común, ya sea a escala global, regional o local. Lo anterior, uniendo también el conocimiento ancestral y los diversos saberes de los locales sobre la biodiversidad que los rodea.

Por último, vale la pena rescatar lo dicho por González (2002), quien afirma:

\footnotetext{
"la gestión de la biodiversidad es un asunto complejo y no se encuentra sólo en manos de los expertos, sino que requiere de la movilización de las personas precisas en cada caso, a nivel individual y colectivo. Es necesario un cambio social para conseguir un cambio en las tendencias de pérdida de la biodiversidad. Pero muchos sectores y grupos implicados en las estrategias de biodiversidad aún no se percatan de la importancia del cambio social, ni del papel que puede desempeñar la educación" (p. 76).
}

Lo anterior se puede complementar con lo encontrado por MathezStiefel et al. (2019) en Madre de Dios, Perú, quienes concluyen que debe existir una integración entre la ciencia y la sociedad, para lo cual se hace 
necesario identificar todos los actores sociales y conocer sus percepciones sobre la biodiversidad del sitio que habitan; sólo así es posible entender la complejidad que se presenta en torno a la biodiversidad y los determinantes que la afectan, para así poder implementar estrategias alternativas que aseguren la conservación de la biodiversidad y el bienestar que ésta ofrece a las personas.

Todo esto sigue en concordancia con lo planteado en el presente documento, reafirmando que a partir del conocimiento de los diferentes conceptos, teorías y procesos que implica la biodiversidad, incluyendo los determinantes de ésta, pueden ser usados como base de la educación ambiental para propender por su protección y conservación por parte de los diferentes actores sociales.

Por último, es preciso analizar algunos beneficios intrínsecos de la conservación de la biodiversidad, los cuales no solo son ecológicos, sino también económicos. Para los primeros, se puede tomar como ejemplo los páramos. Se sabe que el país contiene aproximadamente el $50 \%$ de este ecosistema a nivel mundial; los páramos, además de presentar una alta diversidad de especies, (10\% de la biodiversidad del país), también ofrecen servicios a la población como almacenamiento de carbono atmosférico y regulación hídrica (Vergara-Buitrago, 2020). En ese orden de ideas, Colombia ha hecho grandes esfuerzos por la conservación de páramos, a tal punto que actualmente el $51,7 \%$ de éstos se encuentra protegido legalmente. Aunque dichos esfuerzos son significativos, continúan siendo insuficientes y no se debe olvidar que al promover la conservación de los páramos se genera una oportunidad de protección del $10 \%$ de la biodiversidad del país, a medida que se asegura el abastecimiento del recurso hídrico en ciudades capitales como Bogotá y Bucaramanga.

En cuanto a los beneficios económicos de la conservación de ecosistemas colombianos, se sabe que una hectárea de bosque seco tropical puede capturar más de 57 toneladas de $\mathrm{CO}_{2}$. Teniendo en cuenta que el valor de referencia por tonelada de $\mathrm{CO}_{2}$ capturada equivale a 11,13 dólares, un estudio en El Carmen de Bolívar evidenció que una zona en buen estado de conservación ofrecería aproximadamente 637,22 dólares por hectárea, traducido en un valor total de317480 dólares para las 353 hectáreas del área estudiada (Zuluaga-Zuluaga y Castro-Escobar, 2018). Todo lo anterior es posible debido a la biodiversidad vegetal característica 
de estos ecosistemas, por lo que nuevamente se resalta su importancia, así como la necesidad de entender que el concepto de biodiversidad puede incluir el componente económico para la población humana.

Finalmente, a partir de todo lo anterior es posible inferir que mediante la generación de conocimiento sobre la biodiversidad en estos ecosistemas colombianos, se obtienen insumos necesarios para posicionarla como un referente esencial de la conservación en Colombia, la cual urge en los escenarios actuales de detrimento ecosistémico, y no se debe olvidar que, en este contexto se hace relevante incluirla en las estrategias de Educación Ambiental promovidas por la normatividad vigente y la academia desde diferentes disciplinas.

\section{Conclusiones}

Desde hace más de una década se viene reportando un deterioro gradual de la biodiversidad en Colombia, siendo los ecosistemas de bosque seco tropical, bosque húmedo tropical, páramos y arrecifes de corales, los más amenazados por actividades antropogénicas. Por tratarse de una situación persistente y que avanza con rapidez, son diversas las estrategias que se han sugerido e implementado para mitigar o detener el daño causado por la intervención humana. En este sentido, la Educación Ambiental se ha propuesto como el pilar para transformar hábitos que impactan negativamente la biodiversidad de esos esos ecosistemas. Por ejemplo, existe una política nacional de educación ambiental (PNEA) en el país; no obstante, ésta parece ser insuficiente en sus estrategias para transformar esos hábitos y culturas que van en contravía de la conservación de estos ecosistemas amenazados. Es por lo anterior que entender la biodiversidad como la esencia de la vida en los ecosistemas, así como los mecanismos que han actuado durante millones de años para generar la gran variedad de especies que hoy conocemos, podría considerarse como el referente fundamental para potenciar esa educación ambiental en el país.

Finalmente, bajo la premisa de que es evidente la desconexión entre naturaleza y sociedad, se propone entonces la biodiversidad como un referente que permita disminuir la brecha ya existente. Tal vez entendiendo esa biodiversidad en su integralidad (no solo un número de especies por área) podría contribuir a cambiar el rumbo de la conservación de ecosistemas degradados como los ya mencionados, y adoptarse como eje central en la educación ambiental en Colombia. 


\section{Referencias bibliográficas}

Arciniega, E. \& Torres, W. (2007). Los programas de comunicación y educación ambiental en la reserva de la biósfera Galápagos, Ecuador. En: Araya Rosas, P. \& Clusener- Godt, M. (Eds.) (2007). Reservas de la biosfera, un espacio para la integración de conservación y desarrollo: experiencias exitosas en Iberoamérica, París: Unesco.

Arbeláez-Cortés, E. 2013. Knowledge of Colombian biodiversity: published and indexed. Biodiversity and Conservation, 22(12), pp. 2875-2906.

Aguilera, M., \& Silva, J. F. (1997). Especies y biodiversidad. Interciencia, 22(6), pp. 299-306.

Andrade, M. G. (2011). Estado del conocimiento de la biodiversidad en Colombia y sus amenazas. Consideraciones para fortalecer la interacción cienciapolítica. Revista de la Academia Colombiana de Ciencias Exactas, Físicas y Naturales, 35(137), pp. 491-508.

Bashir, I., Lone, F. A., Bhat, R. A., Mir, S. A., Dar, Z. A., \& Dar, S. A. (2020). Concerns and threats of contamination on aquatic ecosystems. En: Hakeem, K., Bhat, B., Qadri, H. (Eds.). Bioremediation and Biotechnology (pp. 1-26). Cham: Springer.

Bennett, K.D. (1997). Evolution and ecology. Cambridge: Cambridge University Press.

Cruz-García, G. S., \& Peters, P. J. (2015). Conservation of Corals in the Colombian Caribbean. En: Narchi, N., Price, L (Eds.). Ethnobiology of Corals and Coral Reefs (pp. 209-234). Cham: Springer.

Correa, C., Etter, A., Díaz-Timoté, J., Buriticá, S., Ramírez, W., \& Corzo, G. (2020). Spatiotemporal evaluation of the human footprint in Colombia: Four decades of anthropic impact in highly biodiverse ecosystems. Ecological Indicators, 117, 106630.

Frawley, T. H., Finkbeiner, E. M., \& Crowder, L. B. (2019). Environmental and institutional degradation in the globalized economy. Ecology and Society, 24(1), p. 7.

Gaston, K.J. \& Spicer, J. I. (2004). Biodiversity. An Introduction. Oxford: Blackwell Publishing.

González, E. (2002). Educación ambiental para la biodiversidad: reflexiones sobre conceptos y prácticas. Tópicos en Educación Ambiental, 4(11), pp. 76-85.

Halffter, G. (1995). ¿Qué es la biodiversidad?, Butlletí de la Institució Catalana d'Història Natural, 62, pp. 5-14.

Hamilton, A. J. (2005). Species diversity or biodiversity?. Journal of environmental Management, 75(1), pp. 89-92.

Kaennel, M. (1998). Biodiversity: a diversity in definition. En: Bachmann, P., Köhl, M., Päivinen, R (Eds.). Assessment of biodiversity for improved forest planning (pp. 71-81). Dordrecht: Springer 
Martínez, R. (2010). La importancia de la educación ambiental ante la problemática actual. Revista Electrónica Educare, 14(1), pp. 97-111.

Mathez-Stiefel, S., Mulanovich, A., Jaquet, S., Bieri, S., Lojas, J., Breu, T., \& Messerli, P. (2020). Estableciendo una interfaz ciencia-gestión- sociedad para la conservación de la biodiversidad y el bienestar humano en la Amazonia: el caso de Madre de Dios, Perú. Revista cientifica de ecología y medio ambiente, 29(1), p. 1882.

McClanahan, B., Parra, T. S., \& Brisman, A. (2019). Conflicto, ambiente y transición. Colombia, ecología y turismo después de la desmovilización. Crítica Penal y Poder, (16), pp. 99-120.

Martín-López, B., González, J. A., Díaz, S., Castro, I., \& García-Llorente, M. (2007). Biodiversidad y bienestar humano: el papel de la diversidad funcional. Ecosistemas, 16(3), pp. 69-80.

Núñez, I., González-Gaudiano, É., \& Barahona, A. (2003). La biodiversidad: historia y contexto de un concepto. Interciencia, 28(7), pp. 387-393.

Naranjo, L. G. (2019). Imaginarios de naturaleza y el futuro de la biodiversidad. Revista de la Academia Colombiana de Ciencias Exactas, Físicas y Naturales, 43(168), pp. 480-488.

Pérez-Mesa, M. (2019). Concepciones de biodiversidad y prácticas de cuidado de la vida desde una perspectiva cultural. Tecné, Episteme y Didaxis: TED, (45), pp. 17-34.

Rangel, J. O. (2005). La biodiversidad de Colombia. Palimpsestvs, (5), pp. 292304.

Restrepo, J. D., \& Alvarado, E. M. (2011). Assessing major environmental issues in the Caribbean and Pacific coasts of Colombia, South America: An overview of fluvial fluxes, coral reef degradation, and mangrove ecosystems impacted by river diversion. En: Wolanski, E. \& McLusky, D (eds.). Treatise on Estuarine and Coastal Science (pp. 289-314), Waltham: Academic Press

Rull, V. (2004). Biogeografía histórica de las tierras altas de Guayana y origen. Orsis: organismes i sistemes, 19, pp. 37-48.

Saito, C. H. (2013). Environmental education and biodiversity concern: beyond the ecological literacy. American Journal of Agricultural and Biological Sciences, 8(1), pp. 12-27.

Shukla, P. R., Skea, J., Calvo Buendia, E., Masson-Delmotte, V., Pörtner, H. O., Roberts, D. C., ... \& Malley, J. (2019). IPCC, 2019: Climate Change and Land: an IPCC special report on climate change, desertification, land degradation, sustainable land management, food security, and greenhouse gas fluxes in terrestrial ecosystems. Intergovernmental Panel on Climate Chang.

Smith, T. \& Smith R. (2015). Elements of Ecology. Pearson Education.

Soler, M. (2002). Evolución. Granada: Proyecto Sur de Ediciones.

Stupino, S., Iermanó, M. J., Gargoloff, N. A., \& Bonicatto, M. M. (2014). Agroecología: bases teóricas para el diseño y manejo de agroecosistemas 
sustentables. Colección libros de cátedra. La Plata, Argentina: Editorial de la Universidad Nacional de La Plata.

Tan, J., Li, A., Lei, G., Bian, J., \& Zhang, Z. (2019). A novel and direct ecological risk assessment index for environmental degradation based on response curve approach and remotely sensed data. Ecological Indicators, 98, pp. 783-793.

The International Convention on Biological Diversity, 2003. Convention on Biological Diversity: Article 2: Use of Terms. [En línea]: http://www.biodiv. org/ convention/

Van Weelie, D., \& Wals, A. (2002). Making biodiversity meaningful through environmental education. International Journal of science education, 24(11), pp. 1143-1156.

Vergara-Buitrago, P. A. (2020). Estrategias implementadas por el Sistema Nacional de Áreas Protegidas de Colombia para conservar los páramos. Revista de Ciencias Ambientales, 54(1), pp. 167-176.

Yilmaz, M., \& Terzi, F. (2019, February). The Effects of Urban Spatial Development on Coastal Ecosystems: The Case of Mersin, Turkey. In IOP Conference Series: Materials Science and Engineering (Vol. 471, No. 10, p. 102026). IOP Publishing.

Zuluaga-Zuluaga, L., \& Castro-Escobar, E. S. (2018). Valoración de servicios ambientales por captura de $\mathrm{CO} 2$ en un ecosistema de bosque seco tropical en el municipio de El Carmen de Bolívar, Colombia. Revista Luna Azul, (47), pp. 1-20. 